\title{
Effects of Per2 overexpression on growth inhibition and metastasis, and on MTA1, nm23-H1 and the autophagy-associated PI3K/PKB signaling pathway in nude mice xenograft models of ovarian cancer
}

\author{
ZHAOXIA WANG ${ }^{1}$, LI LI $^{1}$ and YANG WANG ${ }^{2}$ \\ ${ }^{1}$ Department of Obstetrics and Gynecology, First Hospital of Shanxi Medical University; \\ ${ }^{2}$ Bank of China Shanxi Branch, Taiyuan, Shanxi 030001, P.R. China
}

Received March 27, 2015; Accepted February 15, 2016

DOI: $10.3892 / \mathrm{mmr} .2016 .5116$

\begin{abstract}
The aim of the present study was to evaluate the association between Period2 (Per2) and the occurrence and development of ovarian cancer, in addition to evaluating the effect of this gene on the growth and metastasis of ovarian cancer in nude mice xenograft models. The detection of Per2 by reverse transcription-quantitative polymerase chain reaction (RT-qPCR) and western blotting methods at various stages of ovarian cancer in tumor tissue samples was conducted. Nude mice xenograft models of ovarian cancer were constructed using an ovarian cancer cell line and, using a gene transfection technique, exogenous infusion of the recombinant gene, Per2, was performed. To assess for the successful and stable expression of Per2 in the tumor tissue, levels of Per2 expression in the nude mice xenograft models were detected by RT-qPCR. During the experimental period, the tumor volumes were measured every three days. Two weeks following treatment cessation, the nude mice were sacrificed and the tumor weight and volume were measured. Furthermore, detection of the changes in expression levels of metastasis-associated gene 1 (MTA-1) and tumor metastasis suppressor gene, non-metastasis protein 23-H1 (nm23-HI), and the expression change of autophagy-associated signal transduction pathway, phosphatidylinositol 3-kinase (PI3K)/protein kinase B (PKB) kinase were analyzed. The findings demonstrated that with ovarian cancer stage development, the expression of Per2 gradually reduced or ceased. In addition, exogenous Per 2 was successfully and stably expressed in nude mice tumor tissue samples.
\end{abstract}

Correspondence to: $\mathrm{Dr} \mathrm{Li} \mathrm{Li}$, Department of Obstetrics and Gynecology, First Hospital of Shanxi Medical University, 85 South Jiefang Road, Taiyuan, Shanxi 030001, P.R. China

E-mail: yaya20120710@163.com

Key words: circadian gene, Period2, gene transfection, ovarian tumor, nude mice xenograft, metastasis-associated gene 1, non-metastasis protein 23-H1, phosphatidylinositol 3-kinase/protein kinase B signaling pathway
Furthermore, in the Per 2 overexpression group, MTA-1 protein expression was significantly reduced when compared with the phosphate-buffered saline (PBS) control and empty plasmid groups, while $\mathrm{nm} 23-\mathrm{Hl}$ protein expression was significantly higher when compared with those two groups. The expression levels of PI3K and PKB kinase, which are marker proteins of the autophagy associated signaling pathway PI3K/PKB, were significantly downregulated, when compared with the PBS control and empty plasmid groups $(\mathrm{P}<0.001)$. Thus, it was demonstrated that Per 2 is closely associated with the development of ovarian cancer, and late-stage ovarian cancer is associated with Per2 mutation or deletion. Per2 overexpression, via exogenous infusion reduced the ovarian cancer growth rate, which was demonstrated by a significant increase in the tumor inhibition rate. In addition, Per2 may inhibit the expression of MTA- 1 and promote the expression of $n m 23-H 1$ to restrict ovarian tumor growth and metastasis. Finally, it is hypothesized that $P e r 2$ affects autophagy by interfering with the PI3K/PKB signaling pathway, causing inhibition of tumor angiogenesis in order to inhibit tumor growth.

\section{Introduction}

Ovarian cancer is a common type of malignant tumor of the female reproductive organs, which is occurring with an increasing incidence (1-4). However, epithelial ovarian cancer, with the highest mortality rate of all types of gynecological tumor, poses a serious threat to the lives of women. As a result of ovarian embryonic development, the tissue anatomy and endocrine function are complex. The tissue anatomy and endocrine function are complex, the early symptoms of ovarian cancer are inconsistent and there are no established primary prevention measures. Surgery performed on patients with epithelial ovarian cancer identified that the tumor, which was confined to the ovary, accounted for only $30 \%$ of the cancer, with the majority spreading to the uterus, bilateral ovaries, omentum and pelvic organs (3). Neither surgery nor chemotherapy is able to achieve a satisfactory outcome. In recent years, with the continuous progression of scientific research, biological therapeutic strategies for ovarian cancer have become a point of interest, and certain studies have 
commenced clinical trials with significant progress (5). Ovarian cancer biotherapy includes gene therapy and immunotherapy. In animal experiments and certain stage I, II and III clinical trials, biotherapy has been effective, and may present as a novel treatment modality following surgery, chemotherapy and radiotherapy $(5,6)$.

In all organisms, the essential biological function is the biological clock. Numerous complex biological functions in vivo are controlled by regulation of the endogenous biological clock, with aspects of behavior and physiology demonstrating a 24-h rhythm (7-9). Casein kinase 1 (CK1), three Period genes (Per1, Per2 and Per3), two Cryptochrome genes (Cryl and Cry2), Clock and Bmall have been identified as core circadian clock genes $(10,11)$. The mammalian $P e r$ genes are key regulators of circadian rhythm.

Previous studies found that biological clock rhythms are associated with cell cycle regulation, and the circadian rhythm system is involved in the cyclic processes of cell growth and apoptosis (12-14). In addition, it has been reported that changes in circadian rhythm are associated with human and other mammalian tumor formation $(15,16)$. Further studies identified that knock down of Per2 impaired the normal circadian rhythms of behavior and physiology, and significantly increased the incidence of the tumor and proliferative phenotype (17-19). These findings demonstrate that Per 2 is important in carcinogenesis. In the study by Fu et al (18), Per2 expression was closely associated with the occurrence of tumors, with the risk of tumor in Per2 mutant mice being significantly higher than that of wild-type mice. The Per 2 mutant mice underwent gamma irradiation and, compared with the wild-type mice, demonstrated increased susceptibility to the gamma rays and a higher risk of cancer (18). Therefore, Per2 maintains normal biological rhythms; however, may also be involved in tumor growth and apoptosis, exerting an inhibitory effect on tumor growth.

As a malignant tumor of the female reproductive system, ovarian tumors are closely associated with hormone secretion. Furthermore, female hormone secretion is closely associated with the circadian rhythm and sleep/wake cycles. At present, and to the best of our knowledge, there are few studies regarding the circadian gene, Per 2 and ovarian cancer. Therefore, in the present study, the changes of Per 2 in ovarian cancer tumor tissue samples at different stages and the possible role of Per2 were analyzed. Nude mice xenograft models of ovarian cancer were used, and Per 2 was overexpressed by exogenous infusion to detect its effect on tumor growth and metastasis, and elucidate the potential underlying mechanism.

\section{Materials and methods}

Specimen source. In order to investigate the change of Per2 during the development of ovarian cancer, archived paraffin-embedded specimens of epithelial ovarian cancer, which had been collected during January 2010 to December 2013 from the First Hospital of Shanxi Medical University (Taiyuan, China), were obtained. None of the patients had received chemotherapy or immunotherapy prior to surgery. The clinical stage was established according to The International Federation of Gynecology and Obstetrics (FIGO) staging system (20); there were eight cases of malignant epithelial ovarian tumor stage (malignant stage) I, eight cases of malignant stage II, and six cases of malignant stage III. Six cases of benign epithelial tumor served as the control group. Written informed consent was obtained from the patients for the paraffin-embedded specimens used in the current study and the animal experiments were approved by the ethics review committee of the First Hospital of Shanxi Medical University. The research was conducted in strict accordance with the provided scheme and relevant provisions of the medical ethics committee of Shanxi Medical University.

Animal and reagents for in vivo research. A total of 28 specific-pathogen-free (SPF), female BALB/c nude mice (body weight, 13-15 g; age, 4 weeks) were obtained from Shanghai Silaike Experimental Animals Co., Ltd. (Shanghai, China). The SK-OV-3 human ovarian cancer cell line was obtained from Peking Union Medical College (Beijing, China). The recombinant plasmid, pcDNA3.1 ${ }^{(+)}-$Per 2 and empty plasmid, pcDNA were obtained from Benyuan Zhengyang Gene Technology Co., Ltd. (Beijing, China). The Plasmid Extraction and Purification kit was purchased from Hangzhou Bioer Technology Co., Ltd. (Beijing, China) and, Invitrogen Lipofectamine 2000 was obtained from Thermo Fisher Scientific, Inc. (Waltham, MA, USA).

Ethics statement. All surgical and animal care procedures were approved by the Medical Ethics Committee of Shanxi Medical University (Shanxi, China). All research was carried out in strict accordance with the guidelines provided by the Medical Ethics Committee.

Cell culture. SK-OV-3 cells were cultured in RPMI-1640 containing $15 \%$ fetal calf serum (Zhejiang Tianhang Biological Technology Co., Ltd., Zhejiang, China), and maintained in an atmosphere of $5 \% \mathrm{CO}_{2}$ at $37^{\circ} \mathrm{C}$. Cells were cultured until the logarithmic growth phase was reached. The cells were harvested, counted and the cell number was adjusted to $2.5 \times 10^{7}$ cells $/ \mathrm{ml}$.

Establishment and grouping of the nude mice xenograft models. The BALB/c nude mice were housed in SPF breeding units. Each nude mice received a subcutaneous injection of $200 \mu 1 \mathrm{SK}-\mathrm{OV}-3$ cell suspension (concentration, $2.5 \times 10^{7}$ cells $/ \mathrm{ml} ; \sim 5 \times 10^{6}$ cells) into the central lateral region of the left axilla. The transplanted tumor had developed after seven days and the tumor diameter was $~ 4-5 \mathrm{~mm} 14$ days after the injection, which indicated successful construction of the model. There were 24 successful mouse models, which were randomly divided into a control group and two experimental groups, the recombinant plasmid group and the empty plasmid group, with eight mice per group.

Gene therapy. Following successful establishment of the animal model, a vernier caliper was used for measurement of the subcutaneous tumor size every 3 days. Gene therapy was initiated when the tumor diameter reached $0.4 \mathrm{~cm}$. Gene transfection was performed using Lipofectamine 2000, which was directly injected into the tumor and at the tumor edges by multi-point injection. The treatment strategies were as follows: i) Control group, local tumor injection once every three days, a total of five times, with $100 \mu 1$ phosphate-buffered saline (PBS) per mouse; ii) empty plasmid group, local tumor 
injection once every three days, a total of five times, with $100 \mu \mathrm{l}$ pcDNA3.1 (100 mg/l) per mouse, in addition to a $25-\mu \mathrm{l}$ injection of Lipofectamine 2000 for transfection. iii) Per2 group, multi-point tumor injection once every three days, a total of five times, of $100 \mu \mathrm{l}$ pcDNA3.1 $1^{(+)}-$Per2 $(100 \mathrm{mg} / \mathrm{l})$ per mouse, in addition to a $25-\mu 1$ injection of Lipofectamine 2000 for transfection. Every three days, vernier caliper measurements of the mouse tumor length (L) and short diameter (W) were taken. Two weeks after treatment cessation the nude mice were sacrificed by cervical dislocation, and the tumor volume $\left[\mathrm{V}\left(\mathrm{mm}^{3}\right)=\mathrm{Lx} \mathrm{W}^{2} / 2\right]$ and weight $(\mathrm{g})$ were measured to calculate the tumor inhibition rate $(\%)$ as follows: $\%=($ control group mean tumor weight - treatment group mean tumor weight) / control group mean tumor weight x 100 .

Reagents. A High Pure FFPE RNA extraction kit (Comwin Biotech Co., Ltd., Beijing, China) was used to extract RNA from paraffin-embedded tissue. In addition, a SYBR Green Real-Time polymerase chain reaction (PCR) kit (Roche Diagnostics, Indianapolis, IN, USA) was used to perform qPCR.

Per2 RNA expression levels in tumor tissue and tumor metastasis-associated gene, MTA-1 and suppressor gene, nm23-H1 in mouse tumors, as detected by reverse transcription-quantitative PCR (RT-qPCR). The RT conditions were as follows: RNA $(11 \mu \mathrm{l} ; 1 \mu \mathrm{g})$ and $1 \mu \mathrm{l}$ random primers $(0.2 \mu \mathrm{g} / \mathrm{ml})$ were incubated at $65^{\circ} \mathrm{C}$ for $5 \mathrm{~min} ; 4 \mu \mathrm{l}$ X Buffer, $3 \mu \mathrm{ldNTP}$ (10 mmol/l), $1 \mu \mathrm{l}$ RNA enzyme inhibitor $(20 \mathrm{U} / \mu \mathrm{l})$ and $1 \mu \mathrm{l}$ reverse transcriptase $(20 \mathrm{U} / \mu \mathrm{l})$ were added [from a RevertAid First Strand cDNA Synthesis Kit (Thermo Fisher Scientific, Inc., Pittsburgh, PA, USA)] and incubated at $25^{\circ} \mathrm{C}$ for $10 \mathrm{~min}$, followed by incubation at $42^{\circ} \mathrm{C}$ for $1 \mathrm{~h}$ and at $72^{\circ} \mathrm{C}$ for $15 \mathrm{~min}$. The qPCR reaction system was as follows: Fast Start Universal SYBR Green Master (ROX; $10 \mu \mathrm{l}$; Roche Diagnostics), $0.5 \mu \mathrm{l}$ upstream primer $(15 \mu \mathrm{M}), 0.5 \mu \mathrm{l}$ downstream primer $(15 \mu \mathrm{M}), 2 \mu \mathrm{l}$ cDNA, $7 \mu \mathrm{l}$ DNAse- and RNase-free water, with a total volume of $20 \mu \mathrm{l}$. The primer sequence for the Per2 gene $\left(G^{2}\right.$ Bank $^{\circledR}$ gene sequence coding AF036893) was as follows: Forward, 5'-GCA GGCTCCACCATGAATGGATACGTGGACTTCT-3' and reverse, 5'-CAAGAAAGCTGGGTGTTACGTCTGGGCCT CTATCCT-3'. The primer sequence for $M T A-1$ was as follows: Forward, 5'-CGCTCAAGTCCTACCTGGAG-3' and reverse, 5'-TGGTACCGGTTTCCTACTCG-3'. The primer sequence for nm23-H1 was as follows: Forward, 5'-ACCTTCATTGCGATC AAACC-3' and reverse, 5'-GGCCCTGAGTGCATGTATTT-3'. The primer sequence for GAPDH was as follows: Forward, 5'-AGAGCTACGAGCTGCCTGAC-3' and reverse, 5'-AGC ACTGTGTTGGCGTACAG-3'. All primer sequences were designed and synthesized by Sangon Biotech Co., Ltd. The PCR reaction conditions were as follows: $94^{\circ} \mathrm{C}$ initial denaturation (10 min); $94^{\circ} \mathrm{C}$ denaturation for $15 \mathrm{sec}$ and $60^{\circ} \mathrm{C}$ annealing and elongation for $60 \mathrm{sec}$, for 40 cycles. Quantitative PCR was conducted to obtain the $\mathrm{Cq}$ value. The difference between the $\mathrm{Cq}$ of the sample determination gene, Per2, and the $\mathrm{Cq}$ of the internal control gene, $\beta$-actin, $(\Delta \mathrm{Cq})$ was calculated as follows: $\Delta \mathrm{Cq}=\mathrm{Cq}_{\mathrm{Per} 2}-\mathrm{Cq}_{\beta \text {-actin }}$ and $\Delta \Delta \mathrm{Cq}$ was obtained by subtracting the $\Delta \mathrm{Cq}_{\text {experimental group }}$ from the $\Delta \mathrm{Cq}_{\text {control group }}$. To determine the expression of Per 2 in the experimental group compared with the normal control group (for multiple sample variation), $2^{-\Delta \Delta \mathrm{Cq}}$ was calculated (21).
Western blot analysis of Per2, MTA-1,nm23-H1,PI3K and PKB kinase protein expression levels. The tumor tissue protein was extracted from paraffin-embedded tissue (Per2) or nude mice with ovarian cancer xenografts (MTA-1, nm23-H1, PI3K and $P K B$ ), using RIPA buffer (Beyotime Institute of Biotechnology, Haimen, China) and the concentration was determined using a bicinchoninic acid assay (Pierce Biotechnology, Inc., Rockford, IL, USA). Proteins (at $10 \mu \mathrm{g} / \mu \mathrm{l}$ ) were separated on a $12 \%$ sodium dodecyl sulfate-polyacrylamide gel by electrophoresis, and transferred onto a polyvinylidene fluoride membrane (Sigma-Aldrich, St. Louis, MO, USA). The membranes were incubated with antibodies to visualize the proteins. These were metastasis-associated gene 1 (MTA-1; cat. no. sc-9445; goat polyclonal), non-metastasis protein 23-H1 (nm23-H1; cat. no. sc-343; rabbit polyclonal), Per2 (cat. no. sc-25363; rabbit polyclonal), phosphatidylinositol 3-kinase (PI3K; cat. no. sc-8010; mouse monoclonal), protein kinase B (PKB) kinase (cat. no. sc-17766) (all 1:500 dilution) and $\beta$-actin (cat. no. sc-47778; mouse monoclonal; reference gene; dilution, 1:1,000) antibodies. Membranes were then incubated with the secondary antibodies bovine anti-goat immunoglobulin $\mathrm{G}$ (IgG)-horseradish peroxidase (HRP) (cat. no. sc-2378) and bovine anti-rabbit IgG-HRP (cat. no. sc-2379) (both used at a 1:2,000 dilution). All antibodies were all purchased from Santa Cruz Biotechnology, Inc. (Heidelberg, Germany). Proteins were detected with enhanced chemiluminescence (ECL) using Pierce ECL Western Blotting substrate (Pierce Biotechnology, Inc.). Analysis of the immune response and exposure were conducted using Image Lab imaging software version 5.2 (Bio-Rad Laboratories, Inc., Hercules, CA, USA). This experiment was conducted five times.

Statistical analysis. SPSS version 16.0 statistical software (SPSS, Inc., Chicago, IL, USA) was used for statistical analysis. Multi-group comparisons were performed using analysis of variance, following a Kolmogorov-Smirnov normality test. Bonferroni's test was used for comparisons between two groups. Data are presented as mean \pm standard deviation. A $\mathrm{P}$-value $<0.05$ was considered to indicate a statistically significant difference.

\section{Results}

Per2 expression level varies at different tumor stages. The expression of Per2 RNA in tumor tissue samples was detected by RT-qPCR. The expression levels of Per2 RNA in the benign tumor group were identified to be significantly greater when compared with the three other groups $(\mathrm{P}<0.001)$. Furthermore, the malignant stage I group demonstrated significantly higher Per2 RNA expression levels versus the malignant stage II and III groups $(\mathrm{P}<0.001)$. No significant difference was identified between the malignant stage II and III groups. The RNA expression level of Per 2 in the benign tumor group was $\sim 14$ times that of malignant stage II group and $\sim 15$ times that of the malignant stage III group (Fig. 1).

Per2 protein expression in tumor tissue samples was detected by western blotting. The expression levels of Per2 at different malignant tumor stages were varied; as the degree of tumor malignancy increased, the Per2 expression gradually 


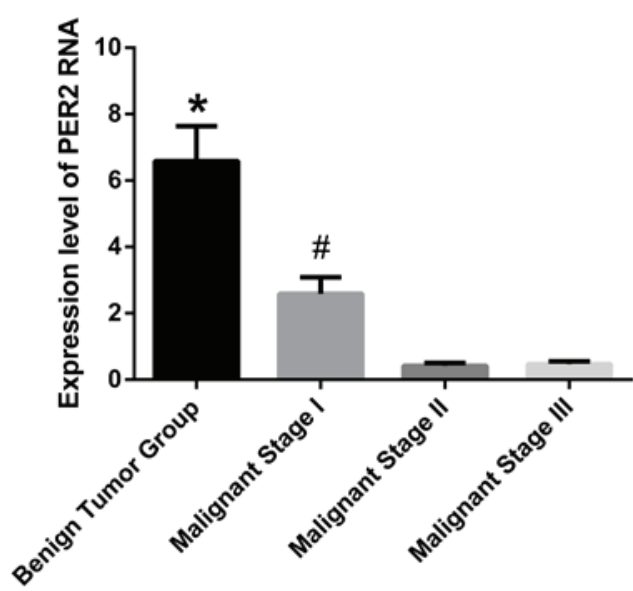

Figure 1. Expression level of Per2 RNA in tumor tissue samples. Data are presented as mean \pm standard deviation. ${ }^{*} \mathrm{P}<0.001$ vs. malignant stages I-III ${ }^{\#} \mathrm{P}<0.001$ vs. malignant stages II and III. Per2, Period2.

decreased (single factor variance analysis between the four groups: $\mathrm{F}=1,785 ; \mathrm{P}<0.001)$. No significant difference was identified between the benign tumor group and the malignant stage I group. A significant difference was observed between the malignant stage I group, and malignant stage II and III groups $(\mathrm{P}<0.05)$, in addition, the difference between malignant stage II and III was significant $(\mathrm{P}<0.05$; Fig. 2).

Per2 overexpression exerts inhibitory effects on ovarian cancer in nude mice xenograft models. Per2 was transfected and successfully expressed in tumor tissue samples. In order to confirm that the liposome-mediated Per2 had been converted into a functional Per2 gene in the body, a specific gene was designed and the expression of Per2 protein was detected by western blot analysis.

No statistically significant difference was identified between the PBS control group and the empty plasmid group; however, the Per2 expression level in the transfected group was significantly greater compared with the other two groups, $(\mathrm{P}<0.01)$. The Per 2 expression level was $\sim 11$ times greater compared with the other two groups (Fig. 3).

Comparison between tumor growth and tumor inhibition. Three mice from the PBS control group and two mice from the empty plasmid group exhibited ascites. The mice with ascites lost weight, consumed less water and presented with cancer-associated cachexia. Furthermore, axillary and inguinal regions in the control and empty plasmid group mice demonstrated varying lymph node sizes and metastasis, and a range of sizes of satellite foci were apparent surrounding the transplanted tumor. In the treatment group overexpressing Per2, ascites and metastasis were not obvious, and visible satellite foci were apparent in one of the mice (data not shown).

The interaction between experiment duration and group was determined and the results are presented in Table I $(\mathrm{F}=22.72 ; \mathrm{P}<0.001)$. The tumor volumes were observed to increase in a time-dependent manner. Upon cessation of treatment, the mean tumor weight of mice from the recombinant
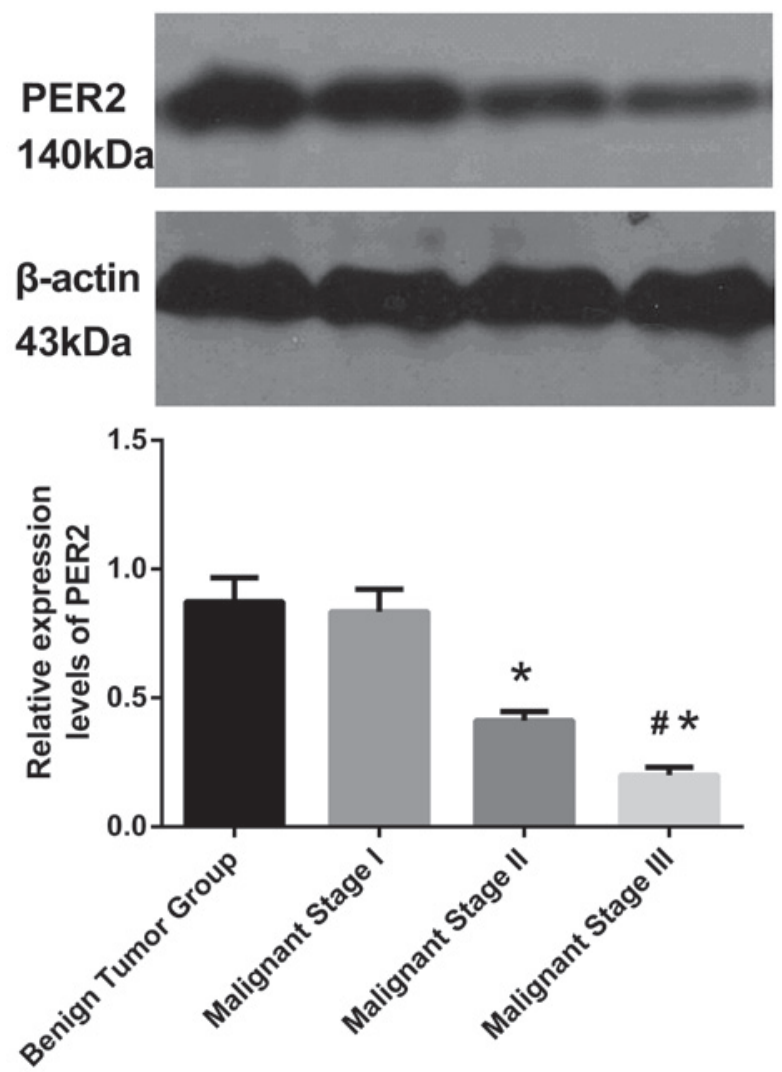

Figure 2. Protein expression level of Per2 in tumor tissue samples. The single factor variance analyses demonstrate statistically significant differences between the four groups $(\mathrm{F}=1,785 ; \mathrm{P}<0.001$, multiple comparisons). Data are presented as mean \pm standard deviation. ${ }^{*} \mathrm{P}<0.05$ vs. benign tumor and malignant stage I; ${ }^{\text {P }}<0.05$ vs. malignant stage II. Per2, Period2.

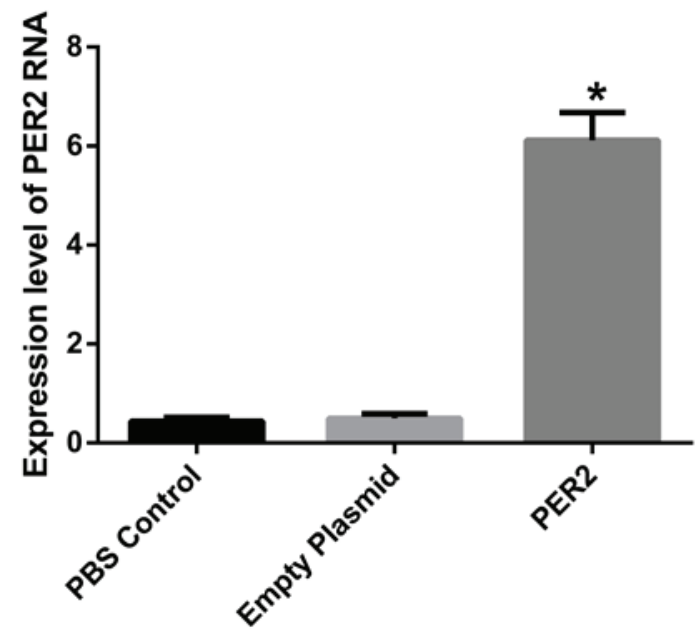

Figure 3. Per2 RNA expression levels in the different treatment groups. Games-Howell correction test of the multiple comparisons was performed. Data are presented as mean \pm standard deviation. ${ }^{*} \mathrm{P}<0.01$ vs. PBS control and empty plasmid. PBS, phosphate-buffered saline.

Per2 plasmid group was significantly less than that of the control and empty plasmid groups $(\mathrm{F}=26.70 ; \mathrm{P}<0.001)$.

Following treatment the mean tumor weights of nude mice were $4.73 \pm 0.95,4.55 \pm 1.06$ and $2.7 \pm 0.52 \mathrm{~g}$, in the control, empty plasmid and Per2 groups, respectively. The tumor weights of 
Table I. Change in tumor volume prior to and following treatment.

\begin{tabular}{lcc}
\hline & \multicolumn{2}{c}{ Volume $\left(\mathrm{mm}^{3}\right)$} \\
\cline { 2 - 3 } Group & Initial & Final \\
\hline PBS control & $72.56 \pm 6.24$ & $845.53 \pm 110.00$ \\
Empty plasmid & $72.49 \pm 7.91$ & $835.32 \pm 105.68^{\mathrm{a}}$ \\
Per2 & $71.24 \pm 5.73$ & $486.38 \pm 70.12^{\mathrm{b}}$
\end{tabular}

Comparison upon measurement of tumors every 3 days, and between the different groups $(\mathrm{F}=22.72 ; \mathrm{P}<0.001)$. With the progression of time, the amount of change observed in tumor volume differed between groups. Following treatment, the tumor volume of the Per 2 recombinant plasmid group is significantly less than that of the control and empty plasmid groups ( $\mathrm{F}=26.699 ; \mathrm{P}<0.001) .{ }^{\mathrm{a}} \mathrm{P}<0.001$ vs. Per2; ${ }^{\mathrm{b}} \mathrm{P}<0.001$ vs. control and empty plasmid. Values are presented as the mean \pm standard deviation. PBS, phosphate-buffered saline; Per2, Period2.

mice in the Per2 group were significantly lighter than those of the control and empty plasmid groups $(\mathrm{P}<0.05)$. In addition, the inhibitory rate of the Per2 group was $42.9 \%$, a significant increase compared with the other two groups (Table II).

RT-qPCR detection of MTA-1 and nm23H1 RNA expression levels. No significant difference was identified between the control and the empty plasmid groups; however, the Per 2 transfected group was significantly different when compared with the other two groups $(\mathrm{P}<0.001)$. In the Per 2 overexpression group, the MTA- $l$ RNA expression was significantly decreased ( $\sim 1 / 10$ of the RNA expression of the PBS control group). However, the RNA expression of $n m 23-H 1$ was increased by $\sim 12$ times when compared with the control group (Fig. 4).

Western blot detection of MTA-1 and nm23-H1 proteins, and protein expression levels of PI3K/PKB pathway marker genes, $P I 3 K$ and $P K B$ kinase. In the $P e r 2$ group, MTA-1 protein expression was significantly downregulated $(\mathrm{P}<0.05$ vs. the other two groups), while the expression level of $\mathrm{nm} 23-\mathrm{H} 1$ was significantly increased ( $\mathrm{P}<0.05$; Fig. 5). The PI3K/PKB signaling pathway marker genes, PI3K and PKB kinase, demonstrated significantly reduced expression levels when compared with the PBS control and empty plasmid groups ( $\mathrm{P}<0.05$; Fig. 6).

\section{Discussion}

In North America, the number of mortalities associated with ovarian cancer is greater than the number of deaths from all other gynecological malignancies $(1,2)$. As the early clinical manifestations of ovarian cancer are not typical and patients do not exhibit obvious discomfort, diagnosis is often late, with symptom such as ascites already apparent, which leads to tumor metastasis. Although the combined treatment of cytoreductive surgery and chemotherapy for ovarian cancer patients initially demonstrates a positive effect, the majority of these patients experience recurrence. The increasing resistance of patients to chemotherapeutic agents, caused by genetic
Table II. Mean tumor weight and tumor inhibition rate.

\begin{tabular}{lcc}
\hline Group & $\begin{array}{c}\text { Tumor weight after } \\
\text { transfection, } g\end{array}$ & $\begin{array}{c}\text { Tumor inhibition } \\
\text { rate, } \%\end{array}$ \\
\hline PBS control & $4.73 \pm 0.95$ & 0.0 \\
Empty plasmid & $4.55 \pm 1.06$ & 3.8 \\
Per2 & $2.7 \pm 0.52$ & $42.9^{\mathrm{a}}$ \\
\hline
\end{tabular}

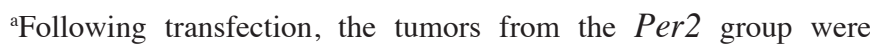
significantly lighter than those of the control and empty plasmid groups $(\mathrm{P}<0.05)$, as assessed by the Student-Newman-Keuls test. Values are presented as the mean \pm standard deviation. PBS, phosphate-buffered saline; Per2, Period2.

instability of tumor cells and a high mutation rate, is the main cause of the high recurrence rate of ovarian cancer, resulting in it becoming an incurable disease. Novel treatment methods, such as gene, immune, interventional and anti-angiogenesis therapy are currently being investigated.

Ovarian cancer is associated with cell proliferation and differentiation of oncogenes and tumor suppressor genes. It is also associated with multi-stage interactions, such as mutation and amplification of ErbB, c-Myc and KRAS oncogenes, and/or of tumor suppressor gene, $R B$, p53 and p16. The loss of function of these genes may lead to the development of tumors. Ovarian cancer is closely associated with the female endocrine system, and the female endocrine system is regulated by circadian rhythm genes; when the rhythm is disrupted, this may cause disorders of the female endocrine system, which may be associated with the occurrence of tumors.

The clock gene, Per2 is important in generating and maintaining circadian rhythms, and is involved in tumor suppression and responding to DNA damage. Per2, via the regulation of oncogenes, tumor suppressor gene expression, and the expression duration of cell cycle regulation-associated genes, inhibits vascular endothelial growth factor (VEGF) agonist activity and, therefore, inhibits tumor development.

Previous studies identified reduced Per 2 expression levels in human colorectal cancer, and that Per2 expression levels were closely associated with patient age, tumor histological grade, invasion depth, lymph node metastasis and TNM staging (22). Recent studies demonstrated that the Per2 protein was expressed in healthy lung tissue samples and non-small cell lung cancer (NSCLC) tissues; however, the expression in NSCLC tissue was significantly reduced. Furthermore, expression of the Per2 protein was found to be associated with the degree of NSCLC differentiation and TNM stage. A lower degree of tumor differentiation and later TNM stage was observed with a reduced expression level of Per2 protein, which demonstrated that the abnormal expression of Per2 in patients with NSCLC was associated with the occurrence and development of NSCLC (23). Yang et al (24) demonstrated that downregulation of Per2 expression accelerated tumor growth, and doubled the diurnal amplitude of the tumor growth rhythm, in an in vitro study. Koyanagi et al (25) indicated that Per2 inhibited VEGF agonist activity, which was induced by tumor cell hypoxia, thereby inhibiting tumor angiogenesis. 


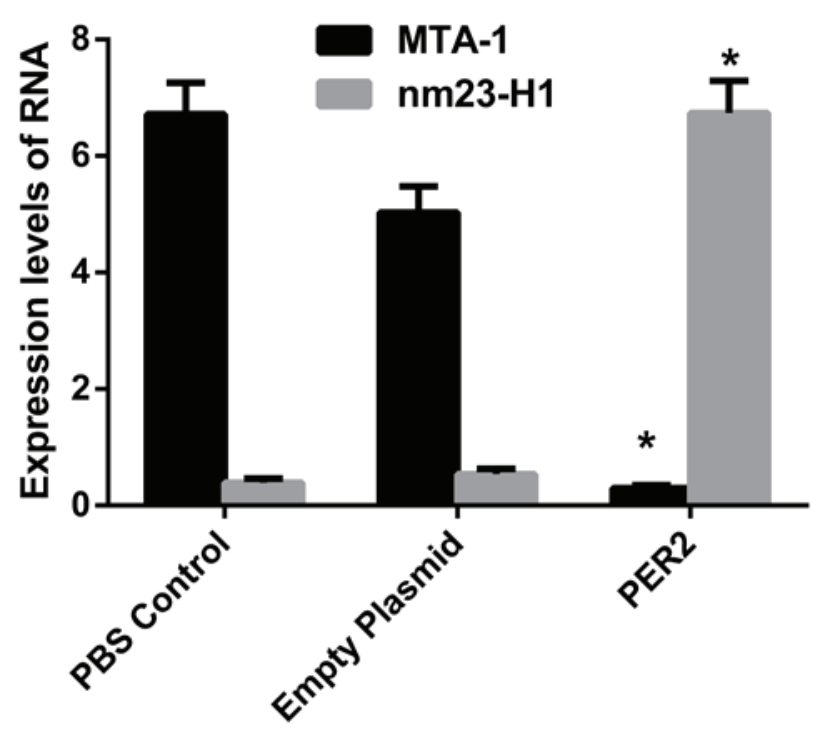

Figure 4. MTA-1 and nm23-H1 RNA expression levels in the different groups. The Bonferroni test was used for multiple comparisons. In the Per2 overexpression group, the MTA-1 expression was significantly decreased, demonstrating an expression level of $\sim 1 / 10$ of the PBS control group. However, the expression level of $n m 23-H 1$ was $\sim 12$ times greater than the PBS control group. Data are presented as the mean \pm standard deviation. ${ }^{*} \mathrm{P}<0.001$ vs. PBS control and empty plasmid. MTA-1, metastasis-associated gene 1; nm23-H1, non-metastasis protein 23-H1; Per2, Period2; PBS, phosphate-buffered saline.
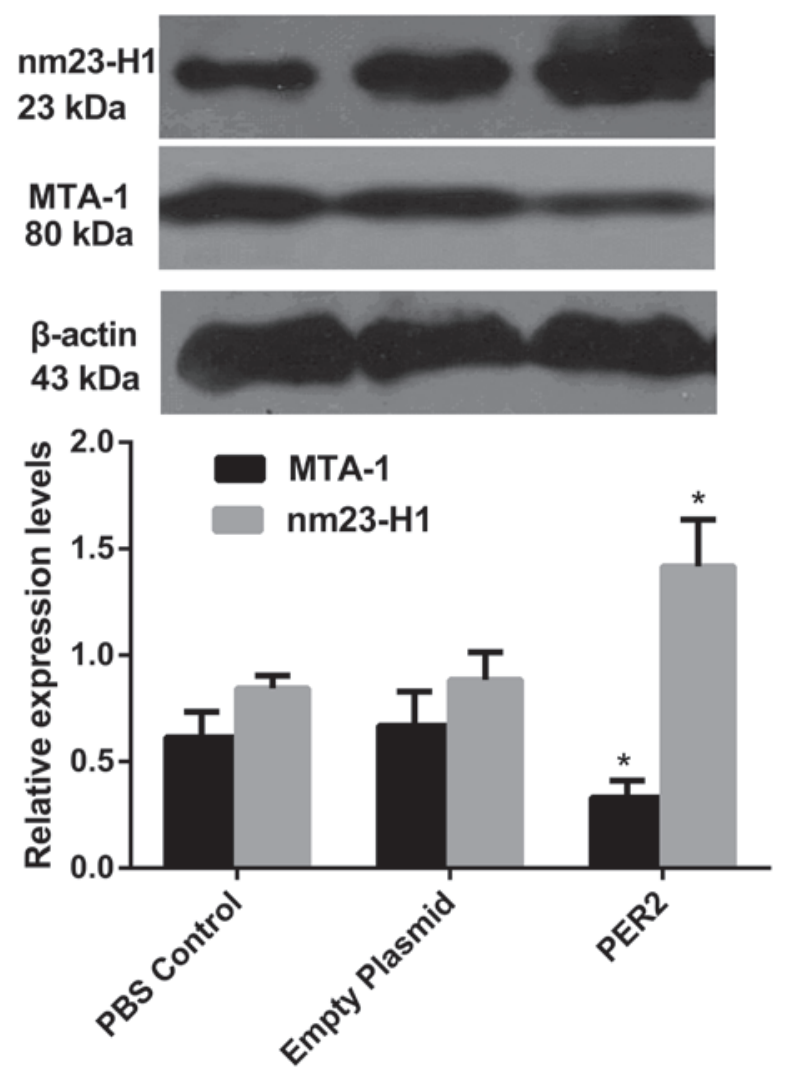

Figure 5. MTA-1 and nm23-H1 protein expression levels in the different groups. MTA-1 protein expression levels in the Per 2 group were significantly downregulated, while the protein expression levels of $\mathrm{nm} 23-\mathrm{H} 1$ were upregulated. Data are presented as the mean \pm standard deviation. ${ }^{~} \mathrm{P}<0.05$ vs. PBS control and empty plasmid. MTA-1, metastasis-associated gene 1; nm23-H1, non-metastasis protein 23-H1; Per2, Period2; PBS, phosphate-buffered saline.

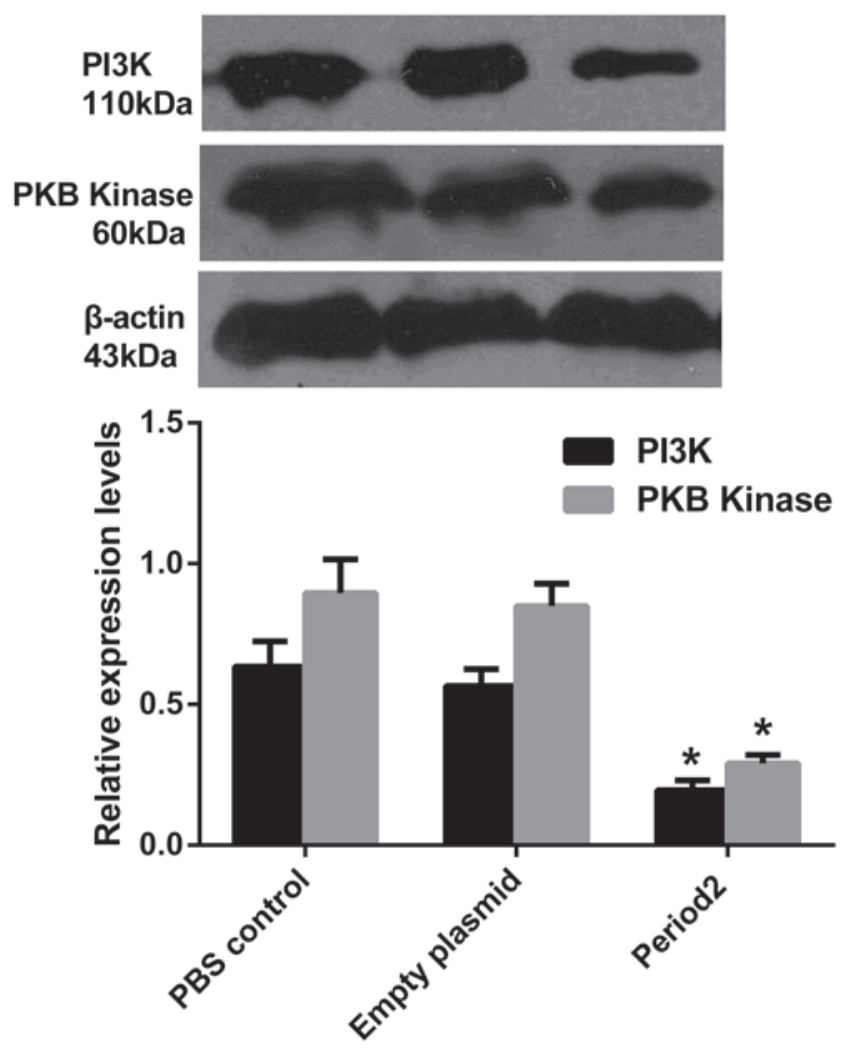

Figure 6. Levels of PI3K and PKB kinase protein expression in the different groups. Data are presented as the mean \pm standard deviation. ${ }^{*} \mathrm{P}<0.05$ vs. PBS control and empty plasmid. PI3K, phosphatidylinositol 3 kinase; PKB, protein kinase B; PBS, phosphate-buffered saline.

MTA-1 was discovered in 1993, demonstrating high expression levels with high metastatic potential in rat breast cancer cells (26). Subsequently, it was confirmed that MTA-1 is involved in the invasion and metastasis of tumors, and is involved in regulating a variety of other malignant tumor processes, including cell proliferation, angiogenesis and resistance to chemotherapy (26-30). Furthermore, MTA-1 is associated with high recurrence and a poor prognosis, and eventually causes cancer. In previous studies, MTA-1 was closely associated with angiogenesis, lymph node metastasis and colorectal and gastric cancers (26,31). In addition, in breast cancer studies, MTA- 1 was correlated with the tumor stage, angiogenesis and recurrence $(32,33)$. In a study regarding small cell lung cancer, it was found that compared with stage I tumors, the MTA- 1 expression level in stage II-IV tumors was significantly increased, and was associated with lymph node metastasis (34). Recently, MTA-1 was identified to be associated with invasive ability, metastasis and a later stage, in oral squamous cell carcinoma (35).

$n m 23$ was the first gene to be identified as a tumor metastasis suppressor gene and two types of $n m 23$ gene are present in humans: $n m 23-H 1$ and $n m 23-H 2$. A close association between nm23-H1 and tumor metastasis has been identified (36). Mutations or low expression levels of $n m 23-H 1$ contribute to tumor metastasis, and its regulation, proliferation and differentiation. Luo et al (37) compared 69 cases of cervical carcinoma and 20 cases of healthy cervical tissue, and the positive expression of $n m 23$ was identified to be negatively correlated 
with the clinical stage, pathological grade and lymph node metastasis $(\mathrm{P}<0.01)$. No association was identified between nm23 expression and the pathological type or patient age. It was hypothesized that reduced levels of $n m 23$ expression may exert a significant effect in cervical carcinogenesis, invasion and transfer, and previous studies have confirmed the association between $\mathrm{nm} 23-\mathrm{Hl}$, and the invasive ability and metastasis of cervical cancer (38). Wu et al (39) demonstrated that the positive expression of $\mathrm{nm} 23-\mathrm{Hl}$ in endometrial carcinoma tissue samples was significantly lower than that of adjacent tissue samples and a healthy uterine mucosa $(\mathrm{P}<0.05)$; the decreased expression of $n m 23-H 1$ in lymph node metastasis $(\mathrm{P}<0.05)$ was not identified to be associated with age, clinical stage or histological grade. Another study demonstrated that the expression of $n m 23-H 1$ mRNA was negatively correlated with prostate cancer bone metastasis and with microvessel density (40).

The PI3K/PKB signaling pathway is a downstream signal transduction pathway involving numerous growth factors and cytokines. The PI3K/PKB signaling pathway is involved in anti-apoptotic activity, and promotes cell proliferation, migration and cancerous transformation processes. Abnormal changes in this pathway, such as increased expression of key oncogenic genes or decreased expression of tumor suppressor genes, occur in certain types of malignant tumor tissue and are closely correlated with tumor progression (41). The structural changes in key molecules of PI3K/PKB, such as pll0 $\alpha$, p85 $\alpha$, $\mathrm{PKB}$ and phosphatase and tensin homolog-encoding genes, are associated with cell transformation, and these genes have been proven to be oncogenes or tumor suppressors, dependent on the gene. The PI3K/PKB signaling pathway is involved in cell signal transduction to regulate apoptosis; previous studies demonstrated that the upregulation of phosphorylated-PKB inhibits apoptosis and is a primary regulatory mechanism of autophagy. PKB-deficient mouse embryonic fibroblast cells demonstrate a reduced rate of progression from the $G_{2} / M$ to the $G_{1}$ phase, indicating that the activation of PKB causes cells to divide rapidly (42). In addition, the PI3K/PKB signaling pathway also affects glucose metabolism and promotes the growth of tumor cells (43). The PI3K/PKB signaling pathway inhibits cell apoptosis, promotes cell proliferation and is associated with tumor occurrence, whilst also affecting migration and invasion of tumor cells, and promoting tumor progression. In a previous study of cell proliferation and migration, the PI3K inhibitor LY294002 eliminated the polar localization of $\mathrm{PKB}$, and reduced the speed of cell migration by $>60 \%$, indicating that cell migration is dependent on the PI3K/PKB signaling pathway (44).

The present study demonstrated that the expression of Per2 in tumor tissue samples varied with each stage of malignant ovarian cancer, when compared with the benign ovarian tissue samples. Furthermore, increases in the degree of tumor differentiation and the TNM stage were associated with a gradual decrease in the expression level of Per2, indicating that the circadian gene is closely associated with the occurrence and development of ovarian cancer. In addition, it was hypothesized that Per2 deletion or mutation leads to tumor progression and metastasis.

In order to verify the possible role of Per2 in ovarian cancer inhibition, a Per 2 recombinant plasmid was transfected into ovarian cancer cells from ovarian xenografts in nude mice. The overexpression of Per2 in the transplantation tumor was stable, and continuous high expression was observed for 4-6 weeks, which was adequate time to allow growth inhibition of the tumor. The tumor growth rate and tumor volume were significantly reduced when compared with the PBS control group, and the tumor inhibition rate increased significantly. Tumor metastasis and the rate of ascites formation was significantly lower than in the PBS control group, with no adverse effects (such as those that are observed with chemotherapy) on survival in the experimental mice. The local and continuous Per 2 expression resulted in a significant decrease in MTA-1 expression, which is highly associated with solid tumor metastasis, and indicates that the tumor metastasis inhibition mechanism of Per2 may act by inhibiting $M T A-1$ expression. In addition, previous studies revealed that the grade and lymph node metastasis in cervical cancer cases were closely and negatively associated with $n m 23-H 1$ expression (45-47). In the present study, the local and continuous elevated expression of Per2 induced the expression of $n m 23-H 1$, which was significantly increased when compared with the PBS control group. Notably, the occurrence and development of tumors is closely associated with the PI3K/PKB signal transduction pathway, which has an important role. The local and continuously elevated expression of Per2 inhibits PKB activation, thus inducing apoptosis of tumor cells, inhibiting the expression of its downstream oncogenes and, therefore, inhibiting tumor growth. However, it must be noted that the use of Per2 gene therapy alone for the treatment of a malignant tumor only inhibits cancer progression and does not completely eradicate it. It is proposed that combining Per2 gene therapy with traditional methods of tumor therapy, such as chemotherapy, may be more efficacious. The expression of Per2 is hypothesized to increase the sensitivity of tumor cells to chemotherapeutic agents via the PI3K/PKB signaling pathway downstream genes, which enhances the therapeutic agent efficacy. Thus, reducing the required dose of chemotherapeutic agent and reducing the side effects.

In conclusion, the present study indicates that Per2 is closely and negatively associated with the occurrence and development of ovarian cancer. Per 2 expression, and the clinical stage and TNM development of ovarian cancer were identified to be correlated. Furthermore, Per 2 may promote expression of the tumor metastasis suppressor gene, $\mathrm{nm} 23-\mathrm{Hl}$ and inhibit MTA-1 expression, and is hypothesized to exert its antitumor effects via the PI3K/PKB signaling pathway and its downstream genes. To the best of our knowledge, this is the first study regarding expression of the circadian gene, Per2 in ovarian cancer. However, the specific underlying mechanisms require further investigation.

\section{References}

1. Nasioudis D, Sisti G, Kanninen TT, Holcomb K, Di Tommaso M, Fambrini $M$ and Witkin SS: Epidemiology and outcomes of squamous ovarian carcinoma; a population-based study. Gynecol Oncol, 2016.

2. Kosary CL: FIGO stage, histology, histologic grade, age and race as prognostic factors in determining survival for cancers of the female gynecological system: An analysis of 1973-87 SEER cases of cancers of the endometrium, cervix, ovary, vulva, and vagina. Semin Surg Oncol 10: 31-46, 1994.

3. Jiang J, Xie W and Cao J: Current situation of the therapies of middle-advanced ovarian cancer. Zhong Liu Yao Xue 3: 416-421, 2013 (In Chinese). 
4. He JR, Gao X and Ren ZF: Global Incidence Patterns of Female Breast and Ovarian Cancers. China Cancer 3: 169-172, 2009.

5. Lou JY, Peng ZL, Zheng Y, Wang H, He B and Wang HJ: Research on human ovarian cancer cell MDR1 gene silenced by siRNA. Sichuan Da Xue Xue Bao Yi Xue Ban 38: 753-755, 2007 (In Chinese)

6. Menendez L, Walker D, Matyunina LV, Dickerson EB, Bowen NJ, Polavarapu N, Benigno BB and McDonald JF: Identification of candidate methylation-responsive genes in ovarian cancer. Mol Cancer 6: 10, 2007.

7. Tei H, Okamura H, Shigeyoshi Y, Fukuhara C, Ozawa R, Hirose M and Sakaki Y: Circadian oscillation of a mammalian homologue of the Drosophila period gene. Nature 389: 512-516, 1997.

8. Shigeyoshi Y, Taguchi K, Yamamoto S, Takekida S, Yan L, Tei H, Moriya T, Shibata S, Loros JJ, Dunlap JC and Okamura H: Light-induced resetting of a mammalian circadian clock is associated with rapid induction of the mPerl transcript. Cell 91: 1043-1053, 1997.

9. Jin X, Shearman LP, Weaver DR, Zylka MJ, de Vries GJ and Reppert SM: A molecular mechanism regulating rhythmic output from the suprachiasmatic circadian clock. Cell 96: 57-68, 1999.

10. Reppert SM and Weaver DR: Molecular analysis of mammalian circadian rhythms. Annu Rev Physiol 63: 647-676, 2001.

11. Young MW and Kay SA: Time zones: A comparative genetics of circadian clocks. Nat Rev Genet 2: 702-715, 2001.

12. Bjarnason GA and Jordan R: Circadian variation of cell proliferation and cell cycle protein expression in man: Clinical implications. Prog Cell Cycle Res 4: 193-206, 2000.

13. Matsuo T, Yamaguchi S, Mitsui S, Emi A, Shimoda F and Okamura H: Control mechanism of the circadian clock for timing of cell division in vivo. Science 302: 255-259, 2003.

14. Panda S, Antoch MP, Miller BH, Su AI, Schook AB, Straume M, Schultz PG, Kay SA, Takahashi JS and Hogenesch JB: Coordinated transcription of key pathways in the mouse by the circadian clock. Cell 109: 307-320, 2002.

15. Barbason H, Herens C, Robaye B, Milis G, Sulon J, Bouzahzah B and VanCantfort J: Importance of cell kinetics rhythmicity for the control of cell proliferation and carcinogenesis in rat liver (review). In Vivo 9: 539-548, 1995

16. Hansen J: Increased breast cancer risk among women who work predominantly at night. Epidemiology 12: 74-77, 2001.

17. Lee CC: Tumor suppression by the mammalian Period genes Cancer Causes Control 17: 525-530, 2006.

18. Fu L, Pelicano H, Liu J, Huang P and Lee C: The circadian gene Period 2 plays an important role in tumor suppression and DNA damage response in vivo. Cell 111: 41-50, 2002.

19. Gery S, Komatsu N, Baldjyan L, Yu A, Koo D and Koeffler HP: The circadian gene perl plays an important role in cell growth and DNA damage control in human cancer cells. Mol Cell 22: 375-382, 2006.

20. Pecorelli S, Benedet JL, Creasman WT and Shepherd JH: FIGO staging of gynecologic cancer. 1994-1997 FIGO committee on Gynecologic Oncology. International Federation of Gynecology and Obstetrics. Int J Gynaecol Obstet 65: 243-249, 1999.

21. Pfaffl MW: A new mathematical model for relative quantification in real-time RT-PCR. Nucleic Acids Res 29: e45, 2001.

22. Karantanos T, Theodoropoulos G, Pektasides D and Gazouli M: Clock genes: Their role in colorectal cancer. World J Gastroenterol 20: 1986-1992, 2014.

23. Chi C, He ZF, Liu Y, Lin XM and Sun CC: Expression and clinical significance of circadian gene Per2 in non-small cell lung cancer. Zhonghua Zhong Liu Za Zhi 35: 129-131, 2013 (In Chinese)

24. Yang X, Wood PA, Oh EY, Du-Quiton J, Ansell CM and Hrushesky WJ: Down regulation of circadian clock gene Period 2 accelerates breast cancer growth by altering its daily growth rhythm. Breast Cancer Res Treat 117: 423-431, 2009.

25. Koyanagi S, Kuramoto Y, Nakagawa H, Aramaki H, Ohdo S, Soeda $\mathrm{S}$ and Shimeno H: A molecular mechanism regulating circadian expression of vascular endothelial growth factor in tumor cells. Cancer Res 63: 7277-7283, 2003

26. Toh Y, Oki E, Oda S, Tokunaga E, Ohno S, Maehara Y, Nicolson GL and Sugimachi K: Overexpression of the MTA1 gene in gastrointestinal carcinomas: Correlation with invasion and metastasis. Int J Cancer 74: 459-463, 1997.

27. Talukder AH, Mishra SK, Mandal M, Balasenthil S, Mehta S, Sahin AA, Barnes CJ and Kumar R: MTA1 interacts with MAT1, a cyclin-dependent kinase-activating kinase complex ring finger factor, and regulates estrogen receptor transactivation functions. J Biol Chem 278: 11676-11685, 2003.
28. Avtanski DB, Nagalingam A, Kuppusamy P, Bonner MY, Arbiser JL, Saxena NK and Sharma D: Honokiol abrogates leptin-induced tumor progression by inhibiting Wnt1-MTA1- $\beta$-catenin signaling axis in a microRNA-34a dependent manner. Oncotarget 6: 16396-16410, 2015.

29. Marzook H, Deivendran S, Kumar R and Pillai MR: Role of MTA1 in head and neck cancers. Cancer Metastasis Rev 33: 953-964, 2014.

30. Bruning A, Blankenstein T, Jückstock J and Mylonas I: Function and regulation of MTA1 and MTA3 in malignancies of the female reproductive system. Cancer Metastasis Rev 33: 943-951, 2014.

31. Deng X, Du L, Wang C, Yang Y, Li J, Liu H, Zhang J, Wang L, Zhang X, Li W, et al: Close association of metastasis-associated protein 1 overexpression with increased angiogenesis and poor survival in patients with histologically node-negative gastric cancer. World J Surg 37: 792-798, 2013.

32. Martin MD, Hilsenbeck SG, Mohsin SK, Hopp TA, Clark GM, Osborne CK, Allred DC and O'Connell P: Breast tumors that overexpress nuclear metastasis-associated 1 (MTA1) protein have high recurrence risks but enhanced responses to systemic therapies. Breast Cancer Res Treat 95: 7-12, 2006.

33. Cheng CW, Liu YF, Yu JC, Wang HW, Ding SL, Hsiung CN, Hsu HM, Shieh JC, Wu PE and Shen CY: Prognostic significance of cyclin D1, $\beta$-catenin, and MTA1 in patients with invasive ductal carcinoma of the breast. Ann Surg Oncol 19: 4129-4139, 2012.

34. Zhang H, Zhu X, Li N, Li D, Sha Z, Zheng X and Wang H: miR-125a-3p targets MTA1 to suppress NSCLC cell proliferation, migration, and invasion. Acta Biochim Biophys Sin 47: 496-503, 2015.

35. Kawasaki G, Yanamoto S, Yoshitomi I, Yamada S and Mizuno A: Overexpression of metastasis-associated MTA1 in oral squamous cell carcinomas: Correlation with metastasis and invasion. Int J Oral Maxillofac Surg 37: 1039-1046, 2008.

36. Gilles AM, Presecan E, Vonica A and Lascu I: Nucleoside diphosphate kinase from human erythrocytes. Structural characterization of the two polypeptide chains responsible for heterogeneity of the hexameric enzyme. J Biol Chem 266: 8784-8789, 1991 .

37. Luo $S$, Wang $X$ and Sun $X$ : The expression and clinical significance of p16 and nm23 in cervical carcinoma. Zhong Guo Shi Yong Fu Ke Yu Chan Ke 7: 421-422, 2004 (In Chinese).

38. Utrera-Barillas D, Salcedo-Vargas M, Gariglio-Vidal P, Hernández-Hernández DM, Gutiérrez-Delgado F and Benítez-Bribiesca L: H-ras and $\mathrm{Nm} 23-\mathrm{H} 1$ gene expression and proteolytic activity in squamous cell carcinoma of the uterine cervix. Arch Med Res 31: 172-181, 2000.

39. $\mathrm{Wu} \mathrm{W}, \mathrm{Xu} \mathrm{H}$ and Deng Z: Expression of nm23H1 and E-cadherin protein in 39 cases of endometrial carcinoma. Zhong Liu Xue Zazhi 4: 322-323, 2006.

40. Ding GF, Li JC and Xu YF: Study on the correlationship between the expression of nm23H1mRNA, TGF-betalmRNA and tumor metastases, survival rate with prostate cancer. Fen Zi Xi Bao Sheng Wu Xue Bao 39: 544-552, 2006 (In Chinese).

41. Nicholson KM and Anderson NG: The protein kinase B/Akt signalling pathway in human malignancy. Cell Signal 14: 381-395, 2002.

42. Li Q and Zhu GD: Targeting serine/threonine protein kinase $\mathrm{B} / \mathrm{Akt}$ and cell-cycle checkpoint kinases for treating cancer. Curr Top Med Chem 2: 939-971, 2002.

43. Elstrom RL, Bauer DE, Buzzai M, Karnauskas R, Harris MH, Plas DR, Zhuang H, Cinalli RM, Alavi A, Rudin CM and Thompso CB: Akt stimulates aerobic glycolysis in cancer cells. Cancer Res 64: 3892-3899, 2004.

44. Kim D, Kim S, Koh H, Yoon SO, Chung AS, Cho KS and Chung J: $\mathrm{Akt} / \mathrm{PKB}$ promotes cancer cell invasion via increased motility and metalloproteinase production. FASEB J 15: 1953-1962, 2001.

45. Huang Y, Cai S and Yu S: Relationship between nm23-H1 expression and lymph node metastasis and prognosis in cervical cancer. Zhonghua Fu Chan Ke Za Zhi 32: 718-721, 1997 (In Chinese).

46. Utrera-Barillas D, Salcedo-Vargas M, Gariglio-Vidal P, Hernández-Hernández DM, Gutiérrez-Delgado $F$ and Benítez-Bribiesca F: H-ras and nm23-H1 gene expression and proteolytic activity in squamous cell carcinoma of the uterine cervix. Arch Med Res 31: 172-181, 2000.

47. Tong Y, Yung LY and Wong YH: Metastasis suppressors nm23H1 and $\mathrm{nm} 23 \mathrm{H} 2$ differentially regulate neoplastic transformation and tumorigenesis. Cancer Lett 361: 207-217, 2015. 